\title{
Faire face à la sécheresse. L'expérience française
}

\author{
Confronting drought. The French experience \\ Guy Bédiot
}

\author{
Agence financière de bassin \\ Seine - Normandie
}

\footnotetext{
L'expérience française, notamment celle de 1976, est analysée. Les acteurs de la lutte sont à la fois les organisations professionnelles touchées par le phénomène, et les pouvoirs publics chargés de l'organisation des dispositifs pour faire face:

- à la sécheresse agricole.

- à l'hydraulicité déficitaire dont s'inquiète EDF,

- à la baisse piézométrique qui concerne aussi bien l'eau potable que l'irrigation

- à la chute des débits d'étiage, accompagnée d'une très forte dégradation de la qualité, et pouvant se conclure par l'assec partiel ou total des cours d'eau.

Les actions entreprises et leurs résultats sont décrits par secteurs d'activité.
}

The French experience, in particular in 1976, is analyzed. The agents involved include both the professional organizations adversely affected by the phenomenon and the government agencies responsible for setting up organizations to cope with

- the drought in farming:

- the deficient stream flow, a cause of concern for E.D.F.

- the piezometric decrease which concerns both drinking water and irrigation:

- the drop in low water flows, accompanied by a very strong degrading of quality and which could end with partial or total drying up of the waterways.

The actions undertaken and their result are described for each sector of activity.
Une sécheresse ne survient pas aussi brutalement qu'une crue. Elle résulte d'un processus fondé essentiellement sur le déficit pluviométrique de durée plus ou moins longue. La sécheresse agricole commence très vite : deux semaines sans pluie suffisent pour provoquer des dommages aux cultures sur sol à faible réserve en eau facilement utilisable par les plantes (R.F.U.). La sévérité d'un étiage est plus longue à se manifester : elle découle d'un bilan déficitaire étalé sur plusieurs mois, voire sur plusieurs années.

Les diverses manifestations de la sécheresse se traduisent par :

- le déficit hydrique des sols avec dessèchement des litières sous forêt et, en période végétative, des cultures et des prairies : c'est la sécheresse agricole;

- l'abaissement des niveaux piézométriques lié à un déficit de recharge des nappes lors de l'hiver précédent. Cet abaissement des nappes est parfois considérable sur les plateaux, et à un degré moindre sur les côteaux. Celui de la nappe alluviale est lié à celui de la rivière;

- la diminution du débit des rivières avec une perte de vitesse d'écoulement d'où une décantation des matières en suspension, et une augmentation de la concentration des matières dissoutes. A l'extrême, il y a l'assec des rivières.
Si la canicule est concomitante de la sécheresse, plusieurs phénomènes interviennent :

- l'augmentation du pouvoir évaporant de l'atmosphère qui agit sur la demande en eau donc sur le bilan hydrique; - l'augmentation de la température de l'eau des rivières, qui entraine un accroissement de la végétation aquatique (prolifération d'algues) et une diminution des teneurs en oxygène dissous d'autant plus importante que la rivière est polluée.

Chacune des caractéristiques de la sécheresse a une incidence particulière sur la vie économique.

En 1976, la sécheresse sévit sur l'Europe Occidentale : elle affecte une grande partie du territoire français. Paradoxalement les régions du midi méditerranéen affectées chaque année par la sécheresse estivale sont très peu touchées par le phénomène. Cette sécheresse constitue une référence grâce aux nombreuses informations collectées au cours de cette période. Elle alimente l'essentiel du propos développé ci-après, même si quelques exemples sont tirés d'autres événements. La mise en place des différents dispositifs de lutte contre la sécheresse est décrite dans une première partie. Puis les différentes actions entreprises durant l'événement sont passées en revue avec leurs résultats. Enfin, les premières réflexions émises à l'issue de la crise sont évoquées en conclusion. 
a. DEFICIT DE L'ECOULEMENT

( 15-05-1976)

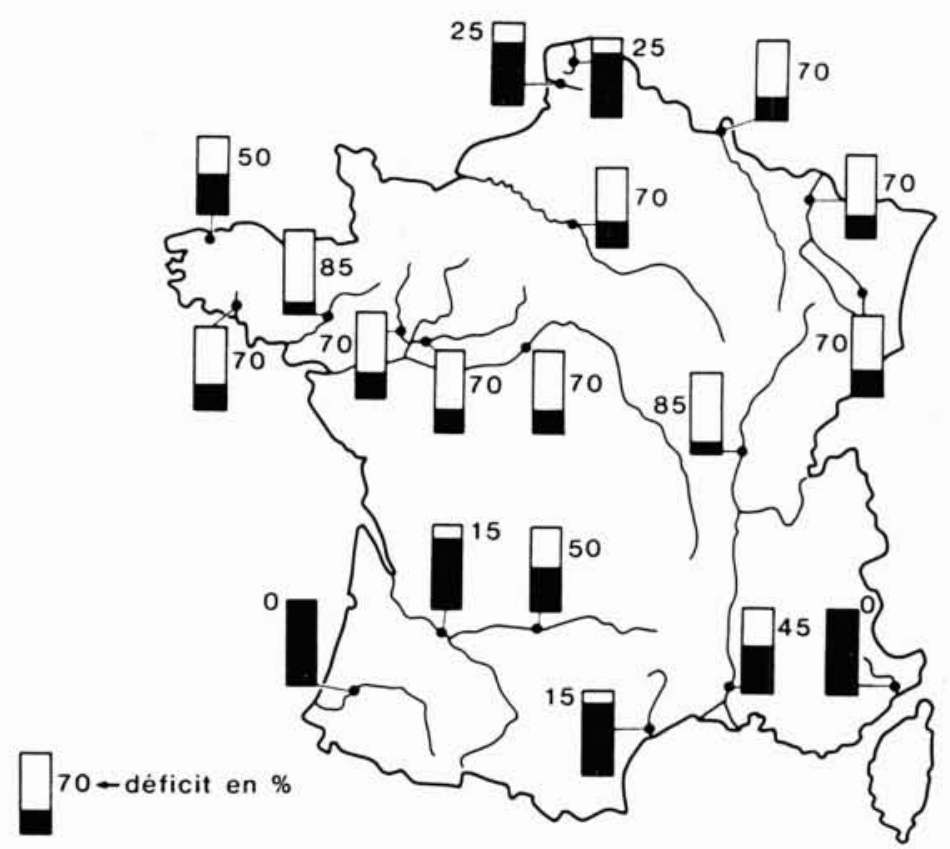
b. DEFICIT DE L'ALIMENTATION DES NAPPES PHREATIQUES
c. DEFICIT DE LA RESERVE EN EAU DU SOL

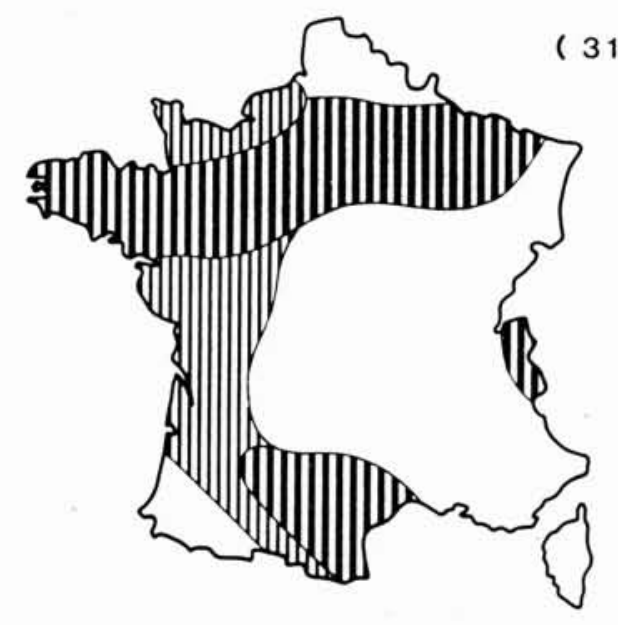

inférieur à $50 \%$

피미 de 50 à $75 \%$

IIIII supérieur à $75 \%$

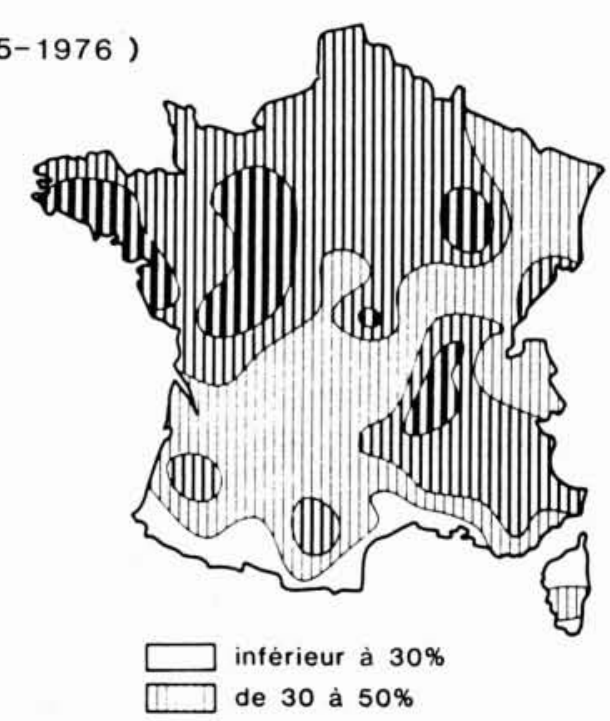

IIIII de 50 à $70 \%$

IIIII supérieur à $70 \%$

Figure 1. - (d'après [1]). 


\section{La mise en place des dispositifs de lutte contre les effets de la sécheresse}

\subsection{La perception du phénomène}

Chronologiquement le phénomène est perçu en premier lieu par Electricité de France, puis par la profession agricole. Le cas de l'administration centrale de l'environnement et celui du Comité technique des barrages-réservoirs de la Seine sont évoqués ensuite.

\subsubsection{Cas de la production hydroélectrique}

A Electricité de France (E.D.F.) la situation est analysée chaque hiver. Dès l'hiver 1975-1976, on sait déjà que le stock nival dont la fonte alimente les grands barrages durant la période de mars à septembre, est très insuffisant. En février 1976, des dispositions sont prises pour utiliser au maximum les groupes thermiques durant les mois à venir. L'objectif est de préserver une partie des réserves réalisées dans les barrages durant le printemps et l'été pour l'utilisation en hiver.

\subsubsection{Cas de la profession agricole}

Dans la presse, les premières traces d'inquiétudes apparaissent au début de la troisième décade d'avril. Les journaux régionaux (« Ouest-France» du 22 avril 1976 : "Paris-Normandie " du 23 avril 1976) évoquent à la fois les préoccupations des exploitants agricoles bretons et normands et l'ampleur de la sécheresse qui sévit déjà en Angleterre.

Les conditions de sécheresse agricole sont relativement fréquentes en France. Elles se manifestent chaque année dans le midi méditerranéen où d'importants aménagements d'irrigation fonctionnent. Dans le Nord de la France, elles entrainent des difficultés plus ou moins aigües une année sur deux pour les différents types de culture. Certaines exploitations sont équipées d'installation par aspersion pour pratiquer des irrigations dites de complément : en principe elles peuvent faire face.

Les exploitations démunies d'irrigation représentent un très fort pourcentage de surfaces cultivées de la France non méditerranéenne auquel il faut ajouter la quasi-totalité des zones d'élevage. Lorsque la sécheresse agricole commence, l'exploitant se trouve dans une position d'attente des prochaines précipitations qui estomperaient ses difficultés. Comme la prévision des précipitations est impossible au-delà de 4 à 5 jours, il prend des dispositions pour assurer l'alimentation et l'abreuvement de son bétail pendant un laps de temps finalement assez court (de quelques jours à quelques semaines) - le temps d'une "soudure" - ou pour pratiquer des cultures dérobées. Une minorité s'équipe en catastrophe avec du matériel d'irrigation.

\subsubsection{Cas de l'administration centrale de l'environne- ment}

Divers services techniques dépendant de différents Ministères sont chargés des réseaux de mesures de pluviométrie, d'hydrométrie, de piézométrie, et de qualité des eaux. L'information est rarement recueillie en temps réel : si l'on excepte les mesures météorologiques connues à une cadence tri-horaire sur la centaine de stations synoptiques et les relevés hydrométriques à cadence journalière sur environ 300 stations en périodes de crues dépassant la cote d'alerte, toute l'information est connue avec un délai de un mois pour les données brutes et de un à trois ans pour les données validées de débits.

En 1976, il n'y a pas de suivi systématique, organisé en temps réel, de la situation hydrologique des rivières et des nappes.

En mai, le service des problèmes de l'eau du secrétariat d'Etat à l'environnement s'inquiète de la situation qui est évoquée le 26 mai en conseil des ministres. Il réunit les avis des experts des différents services concernés dans une note soumise le 2 juin à la Mission interministérielle de l'eau (M.I.D.E.)

A la fin mai le déficit hydrique des sols est très prononcé sur les $3 / 4$ du territoire français (fig. 1 , carte c). Le déficit d'alimentation des nappes d'eau souterraines dépasse les 50 voire les $75 \%$ sur une bande qui va de la Bretagne aux Vosges (fig. 1, carte b). Le déficit d'écoulement des cours d'eau varie de 0 dans le midi de la France à plus de $70 \%$ en Bretagne notamment (fig. 1 , carte a).

La note insiste par ailleurs sur les conséquences à attendre et les difficultés à prévoir sur l'environnement et dans les domaines de l'agriculture et de l'alimentation en eau.

Elle indique en outre les actions à mettre en œuvre : information, mesures préventives, mesures d'urgence.

\subsubsection{Cas des barrages réservoirs du bassin de la Seine}

Lors de sa réunion du 8 juin 1976, le comité technique de l'Institution interdépartementale des barrages réservoirs du bassin de la Seine doit décider du programme de soutien d'étiage des barrages "Seine " et " Marne " situés à plus de 200 kilomètres à l'amont de Paris. Il constate tout d'abord un remplissage incomplet des réservoirs "Seine " (155 $\mathrm{Mm}^{3}$ emmagasinés sur $205 \mathrm{Mm}^{3}$ de capacité totale) et "Marne" (175 $\mathrm{Mm}^{3}$ sur $\left.350 \mathrm{Mm}^{3}\right)^{*}$. Il est démuni d'informations concernant les mesures et les prévisions de débits des rivières, les temps de propagation des lâchures, les volumes prélevés non restitués au cours d'eau et l'évolution future de la demande en eau potable de l'agglomération parisienne dont la fourniture est assurée aux $2 / 3$ par l'eau de rivières. Il prend malgré tout, trois décisions modifiant le règlement d'eau en vigueur :

- début immédiat de la vidange des réservoirs, à une cadence suffisante pour soulager les usines de traitement d'eau potable, malgré le règlement d'eau qui stipule que les lâchures commencent le $1^{\text {er }}$ juillet;

- prise en compte de la période de fermeture des usines au mois d'août en réduisant les lâchures entre le 15 juillet

* Le remplissage du réservoir Marne avait été volontairement limité à la mi-capacité en raison de légers désordres intervenus sur une digue. 
et le 15 août alors que le règlement d'eau prévoit une cadence de vidange identique du $1^{\text {er }}$ juillet au 30 septembre. En l'absence de données, le temps de propagation est estimé à 15 jours. Une étude réalisée en 1980 démontre que le temps de propagation de la lâchure entre le barrage Seine et Paris est de l'ordre de 4 jours !);

- maintien d'une réserve suffisante pour faire face à un étiage qui durerait jusqu'à la mi-décembre comme cela s'était produit en 1971. Là encore, il y a aménagement du règlement d'eau qui prévoit la reprise du remplissage au $1^{\text {er }}$ novembre.

Par ailleurs la vidange décennale du réservoir "Seine " prévue pour novembre 1976 est reportée à l'année suivante.

\subsection{La mise en place des moyens d'action de l'Etat}

\subsubsection{L'expérience du terrain}

Très vite l'administration est confrontée aux problèmes concrets sur le terrain. Ainsi le 11 juin 1976, le Préfet du Morbihan, qui vient de "requérir avec son accord le secours de l'Armée pour assurer un dépannage d'approvisionnement en eau dans une commune, grâce à des camions citernes de l'école de Coetquidan ", pose deux questions au ministère de la Défense, relatives à l'aide de l'Armée :

1) Dans quelles conditions et sous quelles formes juridiques une telle participation peut-elle être envisagée à titre exceptionnel ?

2) Eu égard au fait que ni collectivités locales ni moi-même ne disposons de crédits affectés à ce type d'interventions, dans quelles conditions financières la participation de l'Armée pourrait-elle s'effectuer ? [2].

Cet exemple a le mérite de poser trois types de problèmes sur lesquels le gouvernement doit se prononcer :

- la coordination des moyens des différents services pour organiser la lutte contre les effets de la sécheresse;

- la responsabilité de déclencher l'action aux niveaux local et départemental;

- le financement des mesures d'urgence.

\subsubsection{Les mesures de coordination}

Le 23 juin 1976, une lettre du Premier ministre à Messieurs les ministres et secrétaires d'Etat renforce le rôle de coordination de la Mission interministérielle de l'eau en cette période de sécheresse exceptionnelle.

Dans le courant du mois de juin et au début juillet, un certain nombre de lettres et de circulaires émises par les différents ministères concernés sont envoyées aux préfets et aux responsables de différents services départementaux. Cette correspondance a pour objet :

- d'attirer l'attention sur le déroulement de la sécheresse en cours et de ses effets prévisibles;

- de mettre en place des groupes de travail au niveau départemental pour coordonner les actions des différents services;

- de rappeler les textes règlementaires permettant l'intervention des maires des communes et de l'administration départementale auprès des usagers de l'eau et des orga- nismes socio-professionnels concernés par la sécheresse; - de donner des directives pour faire face de manière opérationnelle à une situation donnée (par exemple : circulaire du 2 juillet du ministère de l'Intérieur concernant les feux de forêts) [3];

- de faciliter la mise à disposition immédiate de crédits de paiement pour des interventions d'urgence;

- d'indiquer les moyens exceptionnels mis à disposition par l'Armée;

- de faire en sorte que les jeunes et les organisations de jeunesse participent à la lutte contre les effets de la sécheresse et que l'ensemble de la population soit tenu informé de la situation et des mesures prises pour y faire face;

- d'autoriser les Préfets à faire des réquisitions de personnes et de matériels en cas de nécessité.

\subsubsection{La responsabilité de l'action}

Les groupes de travail départementaux se constituent vers le 15 juin. Très vite, il est fait usage des pouvoirs de police dévolus aux Maires et aux Préfets.

"Les mesures générales envisagées ou édictées ont porté sur les sujets suivants :

- interdiction d'utiliser l'eau potable pour l'arrosage des jardins d'agrément, le lavage des voitures, le remplissage de piscines privées:

- interdiction des baignades quand la situation l'exige;

- règlementation des pompages dans les rivières avec arrêt des prises d'eau pour l'irrigation si la cote d'alerte est atteinte;

- coupures dans la distribution de l'eau;

- suspension des autorisations de retenues d'eau;

- interdiction de vider les retenues d'eau autorisées;

- interdiction d'arroser avant labour sauf pour les cultu-

res légumières;

- interdiction du brûlage des pailles et chaumes;

- patrouilles de gendarmerie diurnes et nocturnes;

- surveillance accrue des points sensibles des réseaux d'alimentation en eau, des établissements classés, du fonctionnement des stations d'épuration;

- sensibilisation de l'opinion par campagne de presse;

- sensibilisation des industriels par lettre;

- réquisition des moyens de transports et de citernes" $[4$, page 10$]$.

\subsubsection{Le financement des mesures d'urgence}

Quel que soit l'organisme concerné : collectivité locale, département, distributeur d'eau de statut public ou privé, établissement public (E.D.F., Agence financière de bassin), Compagnies d'aménagement, Etat - l'objectif est de dégager rapidement des crédits généralement affectés à d'autres opérations programmées dans l'année en cours et de les rendre disponibles immédiatement. Au début de l'événement, dont la durée et l'ampleur sont inconnues, on ignore quel sera le montant total de la facture à payer. En revanche on sait qu'une approbation de la modification du budget sera nécessaire avec, si possible, de nouvelles recettes pour ne pas altérer le déroulement des opérations en cours sur lesquelles les prélèvements financiers sont effectués.

Ainsi, dans le cas du budget de l'Etat, la loi de finances rectificative pour 1976 est votée par les Assemblées le 29 
octobre 1976. Cette loi entérine la majoration de l'impôt et la levée d'un emprunt obligatoire. C'est le fameux " impôt-sécheresse " qui permet de couvrir les dépenses exceptionnelles de lutte contre les effets de la sécheresse et d'indemnisation des agriculteurs, notamment des éleveurs.

En ce qui concerne les agences de bassin, une lettre du 7 juillet du directeur de la prévention des pollutions et des nuisances, se référant aux textes, lois et décrets en vigueur, demande une participation financière des Agences à la réalisation d'ouvrages permanents nécessités par l'urgence, à l'exclusion des dépenses de fonctionnement. Les agences financières réservent à ce titre $10 \%$ de leurs crédits consacrés au développement des ressources en eau. Chaque subvention accordée à une collectivité locale est calculée sur la base de $15 \%$ du montant des travaux réalisés d'urgence [5].

\subsection{L'organisation du suivi de la situation par une Commission technique interministérielle}

Le suivi de la situation hydrologique et des actions développées sur le terrain est effectué régulièrement au niveau national par un groupe d'experts représentant les différents ministères impliqués dans la lutte contre la sécheresse. C'est cette commission qui élabore les rapports soumis à la Mission interministérielle de l'eau et diffusés ensuite aux responsables départementaux et régionaux [1 à 7].

A l'issue des évènements de 1976, cette commission est maintenue dans son principe. Elle est réunie régulièrement chaque année au début des mois de mars, de juin et septembre. Elle fait le point de la situation hydrologique et de son évolution future. Un bulletin est diffusé.

\section{2. - Les actions entreprises pour faire face aux impacts de la sécheresse et leurs résultats dans différents secteurs}

\subsection{L'alimentation en eau potable}

De janvier à avril 1976, sur les zones du territoire français touchées par le déficit pluviométrique, la demande en eau potable est globalement supérieure de $8 \%$ à celle des mois correspondants de 1975. "A partir des mois de mai et surtout du mois de juin, la demande croît de manière brutale dans toutes les régions, hormis la Côte d'Azur qui bénéficie d'une bonne pluviosité. Les pointes de consommation se produisent plus tôt que d'habitude, présentent généralement une amplitude plus forte et durent beaucoup plus longtemps " [25].

La concomitance de la sécheresse et de la canicule exacerbe l'accroissement des demandes globale et de pointe. (cf. tableaux 1 et 2).

Tableau 1

Pourcentages de variation des volumes distribués mensuellement - Comparison entre 1976 et 1975 (hors région parisienne), d'après M. Dargent [25]

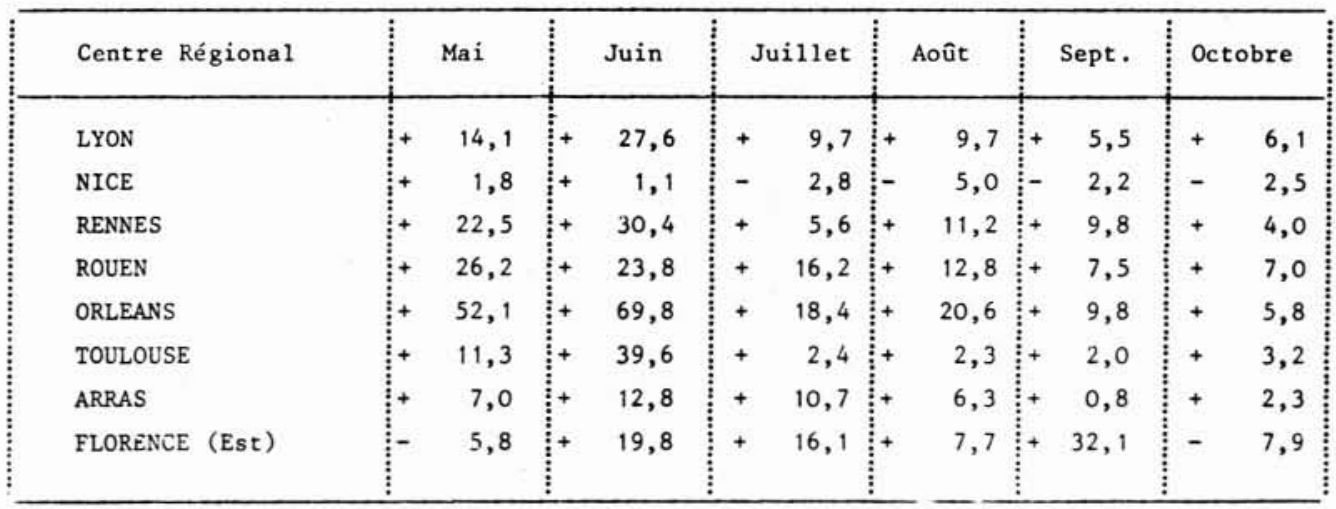

Tableau 2

Coefficient de pointe journalier de production des usines alimentant la ville de Paris intra-muros

\begin{tabular}{|c|c|c|c|c|c|c|c|c|c|c|c|}
\hline ANNEE & 1970 & 1971 & 1972 & 1973 & 1974 & 1975 & 1976 & 1977 & 1978 & 1979 & 1980 \\
\hline COEFFICIENT & 1.18 & 1.16 & 1.10 & 1.19 & $1.11 \vdots$ & 1.13 & 1.25 & 1.18 & 1.16 & 1.19 & 1.18 \\
\hline
\end{tabular}


Dans bien des cas on se trouve en limite des possibilités de production des usines essentiellement pour deux raisons ${ }^{\text {(1) : }}$

- ressource insuffisante;

- mauvaise qualité de l'eau brute et insuffisance des moyens de traitement

Pour faire face à cette situation, quatre types de mesures sont envisagés :

- mesures réglementaires:

- mesures techniques d'urgence pour le maintien d'un service minimal de distribution d'eau;

- accroissement de la ressource disponible avant traitement à l'usine;

- mise en place de traitements supplémentaires pour faire face à la dégradation de la qualité de l'eau brute.

\subsubsection{Les mesures réglementaires}

Il s'agit essentiellement de mesures réglementaires prises au niveau des départements et des communes pour prévenir les défaillances graves du système d'alimentation en eau potable. Déjà évoquées dans les pages précédentes, on les cite à nouveau;

- restriction des prélèvements autres que ceux pour l'eau potable considérée comme besoin prioritaire à satisfaire; - prévention des pollutions en invitant les industriels à limiter leurs rejets et à effectuer une surveillance accrue lors des nettoyages d'usines en période de départs en congé. En dehors de toutes considérations sur l'environnement, il s'agit de préserver les unités de traitement d'eau potable d'une détérioration insurmontable de la qualité de l'eau brute;

- limitation ou interdiction de certains usages de l'eau potable (lavage des voitures, arrosage des pelouses, nettoyage des rues et des caniveaux...). Des appels au civisme sont lancés pour que les abonnés limitent leur consommation.

Dans l'ensemble, ces mesures s'avèrent très efficaces grâce à la prise de conscience des populations sensibilisées par les médias.

\subsubsection{Les mesures techniques d'urgence pour le maintien d'un service minimal de distribution d'eau}

Les coupures d'eau pendant plusieurs heures par jour ont pour effet de réduire la consommation des abonnés et les volumes perdus par fuites dans les réseaux.

L'alimentation par les camions citernes des services de protection civile (véhicules des pompiers) et, à un degré moindre, de certaines unités de l'Armée, est réalisée en attendant que d'autres solutions, provisoires ou durables, soient mises en place. L'armée transporte ainsi $600000 \mathrm{~m}^{3} / \mathrm{km}$ d'eau potable en 1976 [21].

L'interconnexion consiste à raccorder un réseau sous-alimenté à un réseau normalement pourvu. Elle est souvent réalisée par des moyens de fortune (tuyaux souples d'incendie et tuyaux d'irrigation). Un dépannage temporaire avec un raccordement de plusieurs centaines de mètres est un investissement en pure perte. C'est pourquoi, lorsque cela est possible, les équipements sont installés de manière définitive.

\subsubsection{Les mesures techniques d'urgence destinées à accroittre la ressource disponible}

\subsubsection{Eau de rivière: le soutien d'étiage et sa} modulation :

Dans le cas des prélèvements au fil de l'eau, il y a un seuil de débit d'étiage au-dessous duquel la demande n'est plus satisfaite. Le seul ${ }^{(2)}$ moyen d'action - lorsqu'il existe est la vidange des barrages réservoirs situés à l'amont. Les prises d'eau en Marne et en Seine pour l'alimentation en eau de l'agglomération parisienne bénéficient de l'existence des barrages réservoirs à fin multiples (barrages de "Pannessière "; $82 \mathrm{Mm}^{3}$, «Seine ": $205 \mathrm{Mm}^{3}$ et " Marne" $350 \mathrm{Mm}^{3}$ ). "Tout permet de penser que les prélèvements des deux principales usines d'alimentation en eau potable de la Marne (de l'ordre de $10 \mathrm{~m}^{3} / \mathrm{s}$ fin juin sont supérieurs au débit naturel de la rivière, dont le débit n'a été renforcé de façon significative que depuis deux ans grâce à la mise en service du barrage Marne " [25]. Ainsi la décision - prise le 8 juin 1976 - de commencer les lâchures bien avant le $1^{\text {et }}$ juillet porte-t-elle ses fruits, d'autant mieux que le temps de propagation est largement surestimé (cf. § 1.2.4.). Dans les premiers jours de septembre, au moment de la reprise économique, un autre cap difficile est franchi grâce à l'accroissement - sans doute insuffisant - des lâchures effectuées dans la deuxième quinzaine d'août.

\subsubsection{L'eau des nappes souterraines}

a) le cas des puits et forages de plateaux ou de côteaux: Malgré la recharge déficitaire des nappes au cours de I'hiver 1975-1976, la ressource existe en abondance. Cependant, dans de nombreux cas, les puits et forages et/ou leurs équipements s'avèrent insuffisamment profonds et se trouvent dénoyés. Les solutions sont dans l'ordre croissant des coûts correspondants :

- abaissement de la pompe sans approfondissement du puits ou du forage;

- abaissement de la pompe après approfondissement du puits;

- réalisation d'un nouveau forage

b) le cas des nappes alluviales. « Les nappes alluviales sont de plus en plus sollicitées, à la limite de leurs possibilités. Le bilan se trouve déséquilibré lorsqu'on se trouve en présence d'un étiage concomitant d'une canicule qui augmente les besoins " [27].

Une technique de dépannage consiste en la réalimentation de la nappe du champ captant avec de l'eau de rivière. Ce sont les cas d'Albertville sur l'Isère et de Gien sur la Loire cités par Prompsy [27], où la capacité nominale du champ captant est de $6000 \mathrm{~m}^{3} / \mathrm{j}$. A Gien, les puits dans les alluvions sont des ouvrages anciens qui ne recoupent la nappe que sur une hauteur d'environ 3 mètres : ils sont donc particulièrement sensibles aux variations de niveau. Le débit disponible diminue de $5000 \mathrm{~m}^{3} / \mathrm{j}$ le 15 juin à $4000 \mathrm{~m}^{3} / \mathrm{j}$ le 20 , tandis que les demandes

(1) On laisse de côté les cas chroniques de capacité insuffisante de production.

(2) En dehors du recyclage évoqué dans l'exemple de Flavigny-Messein (page 555). 
s'accroissent de 3500 à $5500 \mathrm{~m}^{3} / \mathrm{j}$ dans le même temps.

Deux bassins de $500 \mathrm{~m}^{2}$ sont creusés dans les alluvions à proximité du champ captant et sont ceinturés d'une digue de $2,5 \mathrm{~m}$ constituée des matériaux extraits. On alimente les bassins avec l'eau pompée en Loire à raison de $1000 \mathrm{~m}^{3} / \mathrm{j}$, soit une vitesse d'infiltration maintenue à $1 \mathrm{~m} / \mathrm{j}$. La réalimentation commencée le 4 juillet entraîne une remontée de $0,60 \mathrm{~m}$ sur le forage le plus proche, 24 heures plus tard. Au bout d'une semaine, la capacité du champ captant est rétablie. L'opération s'achève le 10 août.

Un léger traitement d'appoint de chlore à raison de 2 p.p.m. suffit et la filtration dans les alluvions fournit une eau d'excellente qualité [27].

\subsubsection{La mise en place de traitements supplémentaires pour lutter contre la dégradation de la qualité des eaux brutes}

Au cours de la sécheresse de 1976, il n'y a pas détérioration de la qualité des eaux souterraines sauf dans le cas du champ captant de Croissy où la technique de réinjection est utilisée systématiquement depuis la fin des années cinquante : en 1976, $48 \mathrm{Mm}^{3}$ sont pompés dans le gite de Croissy et $30 \mathrm{Mm}^{3}$ d'eau de Seine sont introduits dans les bassins de réalimentation. L'eau de Seine s'est particulièrement dégradée : contrairement à la règle générale, elle est traitée au taux de $240 \mathrm{~g} / \mathrm{m}^{3}$ de sulfate d'alumine (contre 60 en moyenne). Mais surtout son taux d'ammoniaque augmente de $240 \%$ et celui des matières organiques de $85 \%$. L'incidence sur la qualité de l'eau extraite de la nappe est réelle, quoique faible : de 0 en avril à $2,5 \mathrm{mg} / \mathrm{l}$ en novembre 1976 pour retomber à 0 en février 1977 [26].

En revanche la dégradation des eaux brutes des rivières est générale. En Bretagne, dans la région parisienne et dans l'est de la France la teneur en ammoniaque a quasiment triplé. Le DB05 et la concentration des matières oxydables au permanganate augmentent du double [26].

La forte teneur en ammoniaque perturbe l'élimination par la voie biologique dont le système d'aération de l'eau doit être renforcé au coup par coup. Dans certains cas, des unités fonctionnant d'habitude en parallèle sont disposées en série pour renforcer l'épuration biologique: cela se traduit par une baisse importante de la capacité de l'usine.

La filière chimique d'élimination de l'ammoniaque suppose une adaptation souple du taux de traitement de chloration. Mais l'accroissement des teneurs indiqué plus haut a entraîné des problèmes cruciaux d'approvisionnement de réactifs - dont la consommation est 4 à 5 fois supérieure - et de stockage sur place. Par ailleurs, le traitement de teneurs en ammoniaque très fortement variables au moment de la reprise de septembre, entraine la présence de composés chlorés à saveur désagréable qui sont traités au bisulfite de sodium. Par nécessité, le nombre d'analyses de contrôle s'accroît très fortement.

Dans la Seine, de nombreuses algues bleues et des micro-organismes parasites, les actinomycètes, prolifèrent avec l'élévation de température des eaux. Les métabolites issus de leurs activités, communiquent à l'eau brute un goût de moisi caractéristique attribué à la présence de géosmine et d'isobornéol. Pour lutter contre ces saveurs et odeurs, le traitement d'affinage à l'ozone et au charbon actif en poudre ou en grain est systématisé et renforcé [27].
L'ensemble de ces opérations conduit à un surcoût élevé des traitements. L'objectif de maintenir une qualité de l'eau distribuée tout à fait acceptable est malgré tout atteint dans la quasi totalité des cas.

\subsubsection{Eléments d'informations complémentaires con- cernant l'impact sur l'eau potable}

2.1.5.1. Dans le monde rural, près de 7 millions d'habitants sont concernés par la sécheresse de 1976, soit $28 \%$ de la population rurale desservie s'élevant à 25 millions d'habitants sédentaires ou saisonniers.

Les causes des gênes occasionnées se répartissent ainsi :

l'insuffisance des captages des puits (ou pollution) $52 \%$ l'insuffisance des ressources brutes superficielles....16,5\% la saturation des autres ouvrages.................................31,5\%

La moitié des ruraux concernés par la sécheresse subissent des coupures d'eau; $0,7 \%$ reçoivent de l'eau polluée à leur robinet [29].

2.1.5.2. Les conditions climatiques exceptionnelles de l'année 1976 provoquent une éphémère remontée de la consommation d'eau en bouteille qui entre 1973 (3 milliards de litres consommés) et 1977 fléchit d'environ $5 \%$ par an en moyenne.

2.1.5.3. Sur le plan financier, le bilan des interventions d'urgence s'élève à environ 60 millions de francs pour 310 interventions. Le tableau 3 (page suivante) tiré de l'article de Michel Dargent en donne la ventilation par agence de bassin et par rubrique d'intervention. $60 \%$ des interventions coûtent moins de $100000 \mathrm{~F}$ et $9 \%$ plus de $400000 \mathrm{~F}$ [25].

\subsection{L'activité industrielle}

Deux cas sont à considérer : le cas général de l'activité industrielle utilisatrice d'eau et le cas particulier de la production électrique.

\subsection{L'activité industrielle en général}

La plupart des responsables de l'industrie prennent en temps utile des mesures pour assurer la marche, même ralentie, de leurs usines. Hormis les cas rares de stockage d'eau, il est procédé soit à des recyclages d'eau et à toutes mesures d'économie sur les prélèvements, soit à des travaux complémentaires de renforcement d'alimentation. Des usines, notamment les tanneries, ralentissent leur activité ou ferment par insuffisance de débits des rivières conjuguée à un risque accru de dégradation grave de la qualité. D'autres secteurs réduisent leur production par insuffisance d'alimentation en matières premières, par exemple l'industrie agro-alimentaire. Parfois on recourt à une mesure exceptionnelle: ainsi l'alimentation d'une usine de Creusot-Loire est assurée en puisant dans l'étang de Torcy (Saône et Loire) affecté en temps ordinaire aux besoins de la navigation [8].

D'une manière générale les recommandations de l'administration concernant les réductions momentanées des rejets et le contrôle des installations d'épuration sont suivies. En 1976, on ne note pas d'aggravation du nombre de pollutions " accidentelles". 
Tableau 3

Bilan des interventions d'urgence pour assurer l'alimentation en eau potable au cours de la sécheresse 1976 . d'après Michel Dargent [25]

\begin{tabular}{|c|c|c|c|c|c|c|c|c|}
\hline Agences & Interconnexion & $\begin{array}{l}\text { Renforcement } \\
\text { du réseau }\end{array}$ & $\begin{array}{l}\text { Renforcement } \\
\text { de la } \\
\text { ressource } \\
\text { (1) }\end{array}$ & $\begin{array}{l}\text { Renforcement } \\
\text { de la } \\
\text { station de } \\
\text { pompage }\end{array}$ & $\begin{array}{l}\text { Soutien } \\
\text { débit d'étiage } \\
\text { recharge } \\
\text { nappe }\end{array}$ & \begin{tabular}{|c} 
Economie \\
d'eau \\
(industriels)
\end{tabular} & $\begin{array}{l}\text { Nombre total } \\
\text { 'irterventions }\end{array}$ & $\begin{array}{c}\text { Montant des } \\
\text { travaux } \\
\text { réalisés en }\end{array}$ \\
\hline Adour-Garonne & 6 & - & 5 & 1 & 4 & & 16 & 2204053 \\
\hline Artois-Picardie & - & - & - & - & & & - & - \\
\hline Loire-Bretagne & 11 & 13 & 25 & 4 & 1 & 1 & 55 & 28674000 \\
\hline Rhin-Meuse & 11 & - & 20 & 3 & & 1 & 35 & 4379350 \\
\hline $\begin{array}{l}\text { Rhone-Méditerranée } \\
\text { Corse }\end{array}$ & $2 \mathrm{C}$ & 10 & 34 & 6 & . & & 70 & 11451400 \\
\hline Seine-Normandie & 46 & 12 & 59 & 17 & & & 134 & 14140900 \\
\hline TOTAUX & 94 & 35 & 143 & 31 & 5 & 2 & 310 & $60 \quad 849703$ \\
\hline
\end{tabular}

(1) Sous détail :

remise en service d'un forage existant : 19 remise en état d'un captage : 14 réalisation d'un forage réalisation de puits captage de source prise d'eau en rivière
14

47

\subsubsection{La production électrique}

En 1976, la période la plus critique pour la production d'électricité se situe entre le $1^{\text {er }}$ juin et le 15 juillet. La persistance de la sécheresse et la canicule entrainent de très importantes limitations - de $30 \%$ à $50 \%$ - de la production d'origine thermique. Réglementairement, la température limite de l'eau de refroidissement avant rejet à la rivière est de $30^{\circ} \mathrm{C}$. En accord avec les ministères concernés, les centrales thermiques bénéficient d'une dérogation relative à la mesure de la température non pas avant rejet en rivière, mais dans la rivière après mélange. La limite de température est même portée à 31 voire $32^{\circ} \mathrm{C}$, sauf pour les centrales thermiques situées à l'amont d'usines de production d'eau potable. "Malgré ces assouplissements les limitations de production thermique avoisinent certains jours $4000 \mathrm{M}^{\prime} \mathrm{N}$ " [15].

Comme la sécheresse continue d'affecter le secteur hydraulique, les exploitants utilisent alors des moyens de production réservés au moment difficile de l'hiver (turbines à gaz, surcharges de centrales thermiques peu performantes) et recourent à l'achat d'électricité à l'étranger notamment à l'Espagne et à l'Italie qui sont en période de congés. Au total 15 milliards de $\mathrm{kWh}$ supplémentaires sont produits par les centrales thermiques ou achetés à l'étranger [19].

Malgré ce redéploiement, la marge de production reste insuffisante et E.D.F. recourt à deux mesures de restriction :

- la réduction de la tension de distribution de $5 \%$ (courant de basse tension)

- la suspension des contrats dits interruptibles

Fin août les apports hydrauliques sont inférieurs de $30 \%$ à la moyenne. Les pluies reprennent en septembre et tout rentre dans l'ordre pendant le dernier trimestre 1976.
Le coût de l'énergie de remplacement substituée au déficit hydraulique est évalué à environ 1,26 GF (1976).

"Finalement les coupures de courant sont évitées au cours de la sécheresse intense de 1976 grâce à la fois aux dispositions exceptionnelles mises en œuvre en France et à la solidarité étroite qui lie les producteurs d'électricité européens, solidarité qui joue cette fois en faveur de notre pays " [19].

Que se passerait-il en cas de poursuite de la sécheresse en automne? Il est impossible de répondre, mais des éléments peuvent être apportés en analysant l'événement de l'automne 1978 :

Après une année hydrologique 1977-1978 très nettement excédentaire, une sécheresse pluviométrique très sévère survient durant quatre mois de septembre à décembre: les débits des cours d'eau de régime pluvial ou pluvio-nival continuent à baisser, le courant de pointe est produit en turbinant les réserves emmagasinées durant l'été grâce à la fonte des neiges. Ordinairement, Electricité de France passe sans difficulté ce genre d'événements, mais en 1978, le parc de production thermique est fragile : on a abandonné la construction de plusieurs tranches au fuel programmées en 1973 et l'équipement nucléaire a pris du retard. Par ailleurs fin décembre E.D.F., craignant la poursuite de la sécheresse en janvier et février 1979, préfère garder les réserves encore abondantes accumulées derrière les barrages et s'approvisionne en courant produit à l'étranger sans avoir recours à des baisses de tension et à une réduction de la consommation.

La rupture de la principale ligne de transport du courant fourni par l'Allemagne intervient le mardi 19 décembre, et il s'ensuit une panne générale de plusieurs heures, affectant une grande partie du territoire, provoquant des dommages importants. Panne imputable à la sécheresse ? sans doute mais aussi et surtout à la fragilité des équipements d'E.D.F. à ce moment là. 


\subsection{La production agricole}

\subsubsection{L'élevage}

Les ruptures de stock fourrager dans les zones d'élevage sont prévisibles dès la fin avril 1976. Dans une première phase, la profession agricole constitue une réserve fourragère le plus souvent payée par les agriculteurs eux-mêmes avec des facilités d'avance de trésorerie consenties par des organismes bancaires, notamment le Crédit Agricole. Dans une seconde phase, avec l'aggravation du déficit fourrager, des transports de paille sont organisés afin de constituer des rations d'entretien pour les bêtes. Ils sont mis en place avec le concours de l'Armée et de la S.N.C.F. L'Armée mobilise jusqu'à 15000 hommes par jour durant la période critique. Environ 900000 tonnes de paille ou de fourrage sont transportées, ce qui nécessite au total 510000 journées/hommes, 95000 journées/véhicule pour parcourir 15000000 de kilomètres [21]. Avec la canicule, le besoin de boisson des bovins - passant de 40 à 80 litres par jour et par tête - pose d'énormes problèmes, au point que le dispositif de camions citernes ne suffit pas. L'abattage d'une partie du cheptel est l'ultime recours, souvent aggravé par un certain climat de panique. Des solutions transitoires sont trouvées sur place en juillet-août, par exemple en mobilisant les entrepôts frigorifiques des industriels pour éviter que le cours de la viande ne s'effondre. " D'une manière générale, seuls les animaux de réforme et les jeunes bêtes sont abattus; on évite ainsi la décapitalisation du cheptel en préservant les bêtes d'avenir " [13]. La baisse de la production laitière a été sensible : de 15 à $25 \%$ de juin à septembre. Le 15 septembre, en même temps que les premières pluies favorisent une repousse exceptionnelle des herbages, parait le décret instituant une aide exceptionnelle aux éleveurs victimes de la sécheresse. La crise est surmontée.

\subsubsection{Les cultures non irriguées}

Les cultures non irriguées subissent des baisses de rendements de 20 à $45 \%$ pour les céréales d'hiver et de printemps notamment par échaudage. La betterave a un rendement irrégulier et le maïs est souvent fauché en vert pour l'alimentation du bétail. La loi de finances du 29 octobre institue une aide exceptionnelle aux agriculteurs particulièrement atteints par la sécheresse, au titre des calamités.

\subsubsection{Les cultures irriguées}

Les cultures irriguées peuvent se classer en deux catégories. Les unes sont tributaires d'un équipement collectif, et concernent essentiellement les régions du midi de la France, sur des surfaces bien connues avec des doses peu variables d'une année sur l'autre. Les autres dépendent essentiellement de l'initiative individuelle : elles se développent un peu partout, dans le sud ouest et les $2 / 3$ nord de la France, sur des surfaces mal connues avec des doses excessivement variables d'une année à l'autre. Pendant l'événement de 1976, de nombreuses installations pompent à même les rivières souvent avec un équipement très rudimentaire : ce sont les irrigations dites " sauvages " car non déclarées aux administrations habilitées à délivrer les autorisations de prélèvement [10]
Sur le bassin Seine Normandie, J. Carlier note que " les superficies irriguées en 1976 augmentent de $70 \%$ par rapport à celles de 1975. Les volumes prélevés sont multipliés par plus de 3 entre 1975 et 1976. Cette augmentation considérable résulte d'une part de l'augmentation des surfaces irriguées et d'autre part de l'accroissement des volumes par hectare; sur 17 exploitations les volumes par hectares sont en moyenne de $1233 \mathrm{~m}^{3}$ en 1976 contre 619 en $1975^{(3)} \ldots$ La durée de la saison des arrosages double pratiquement, passant de 66 jours ( 9 juin au 15 août) en 1975 à 133 jours ( 26 avril au 9 septembre) en 1976. Les installations d'irrigation (généralement par aspersion) sont utilisées de façon continue, souvent jour et nuit, les seules interruptions de fonctionnement résultant de la nécessité de déplacer les canalisations mobiles et les asperseurs " [13].

« De tels prélèvements opérés dans les cours d'eau ou dans les nappes entraînent fréquemment des difficulés qui se traduisent parfois par des interdictions de poursuivre les prélèvements afin de réserver les faibles ressources disponibles à la satisfaction des besoins prioritaires de l'alimentation humaine ou de l'abreuvement des animaux".

Marcel Mailly indique par ailleurs que " les pompages opérés pour les besoins de l'agriculture soit dans le lit même des cours d'eau soit à proximité immédiate... entrainent en 1976 la mise à sec de nombreuses sections de cours d'eau et s'il est exact que, dans certains départements, des mesures administratives sont prises fort judicieusement pour supprimer ces pompages, dans d'autres, les prélèvements sont poursuivis d'une manière anarchique au détriment des usagers de l'aval " [14].

La forte baisse piézométrique des nappes sur les plateaux et sur les coteaux provoque parfois le dénoyage des pompes installées dans des puits ou dans des forages : des interventions d'urgence sont destinées à approfondir les intallations existantes ou à pratiquer de nouveaux forages. Certains agriculteurs s'équipent en catastrophe avec du matériel d'irrigation qu'ils auront du mal à amortir les années suivantes.

\subsection{L'impact sur l'environnement}

\subsection{1. les incendies de forêts}

La France comporte 14700000 hectares de forêts. Chaque année en moyenne 40000 ha sont la proie des flammes, dont 34000 sur 4700000 ha en zone méditerranéenne (les 14 départements de la zone PROMETHEE ${ }^{(4)}$ régulièrement ravagés par le feu) et 6000 sur 10000000 ha hors zone méditerranéenne.

Chaque année un budget considérable de lutte contre les incendies de forêts est mis en œuvre. En 1982 ce chapitre du budget de l'Etat atteint $350 \mathrm{MF}$ auxquels il faut ajouter $21 \mathrm{MF}$ des collectivités locales. Près de 23000 hommes sont affectés aux incendies de forêts avec des

(3) Des études récentes ont montré que ces valeurs sont vraisemblablement sous-estimées.

(4) La zone PROMETHEE comprend les départements des régions Corse, Provence Côte d'Azur, Languedoc Roussillon, plus le département de l'Ardèche de la région Rhône Alpes [28]. 
moyens comprenant les fameux 12 " canadairs ", capables d'emporter chacun 5,5 tonnes d'eau, et 11 hélicoptères. Outre les efforts faits pour la prévision et la prévention, les services cherchent en permanence à parfaire leur efficacité dans la lutte [33].

En 1976, les feux de forêts gagnent les départements généralement épargnés, mais moins aptes à faire face. Les dégâts sont cependant limités à 109000 ha ${ }^{(5)}$ pour l'ensemble des départements français contre 300000 en 1949 , année sèche comparable [18]. La surface parcourue par le feu dans la zone PROMETHEE reste dans la moyenne : l'accroissement provient essentiellement des autres départements. En plus des moyens de la protection civile, l'Armée met à la disposition des préfets des moyens de surveillance et de prévention des incendies (patrouilles à pied, à cheval, en véhicule terrestres et en avions) et de lutte (15000 hommes/jour, 120 jours/engins spéciaux, 60 heures de vol d'avions légers) [21].

\subsubsection{La mortalité des poissons dans les rivières}

\subsubsection{En rivière non asséchées}

Elle semble due essentiellement à la chute de l'oxygène dissous dans les eaux à la fin du mois de mai 1976, consécutive à une diminution de débit et à un fort réchauffement des eaux. Ce dernier a entraîné une explosion de l'activité photosynthétique des plantes et du phytoplancton qui se traduit par une forte variation diurne du taux d'oxygène dissous (de $5 \mathrm{mg} / 1$ le matin à $10 \mathrm{mg} / \mathrm{l}$ le soir pour les rivières les plus propres, mais 0,2 à 0,5 $\mathrm{mg} / 1$ pour la Seine à Paris) [17].

La diminution du débit entraîne une baisse sensible des vitesses d'écoulement, ce qui permet aux matières en suspension de se déposer et de fermenter. La concentration des matières dissoutes augmente, ce qui accroit le caractère de pollution chronique de certaines rivières. L'ensemble des phénomènes contribuent à la chute du taux d'oxygène dissous et à la mortalité de poissons fin mai et en juin. Par la suite, malgré des débits encore plus faibles au mois d'août, il n'y a pas de mortalité de poissons sauf accident (pollution accidentelle) comme :

- sur le Rhône à Pierre Bénite le 13 juillet : déversement d'acroléine;

- sur le Vézère à Condat le Lardin (Dordogne) le 11 août suite à une mauvaise manœuvre dans une usine de pâte à papier [8];

- sur la Marne à l'aval de Chalons/Marne en juillet... [14].

Avec les premières pluies d'orage de septembre sur la région parisienne, les mortalités de poisson sont à nouveau observées dans la Seine : c'est la conséquence du lessivage des sols urbanisés et du fonctionnement des déversoirs d'orage après de long mois sans pluies importantes.

La seule action urgente possible est le nettoyage des rivières par enlèvement des corps flottants [5].

\subsubsection{Les assecs des cours d'eau}

En 1976 il s'est produit un assèchement de rivières et de ruisseaux généralement considérés comme pérennes : en région Champagne-Ardenne les chiffres sont impressionnants :

\begin{tabular}{|l|c|c|}
\cline { 2 - 3 } \multicolumn{1}{c|}{} & $\begin{array}{c}\text { Complè- } \\
\text { tement } \\
\text { en } \\
\text { assec }\end{array}$ & $\begin{array}{c}\text { Coupures avec } \\
\text { fosses } \\
\text { remplies } \\
\text { d'eau }\end{array}$ \\
\hline $\begin{array}{c}\text { Cours d'eau 1've catégorie } \\
\text { (Salmonidés) }\end{array}$ & $562 \mathrm{~km}$ & $34 \mathrm{~km}$ \\
\hline $\begin{array}{l}\text { Cours d'eau 2" catégorie } \\
\text { (Cyprinidés) }\end{array}$ & $62 \mathrm{~km}$ & $65 \mathrm{~km}$ \\
\hline
\end{tabular}

"Ces renseignements ne concernent que les cours d'eau présentant un intérêt halieutique certain. Mais il faut également tenir compte de très nombreux ruisseaux dans lesquels on ne pêche pas mais où des frayères naturelles importantes sont localisées chaque année. On peut estimer que ce " réseau de reproduction ", qui est complètement anéanti, a une longueur sensiblement égale à celui du réseau dit " de pêche "... " toute la petite faune et la flore se trouvent irrémédiablement détruites sur les parcours asséchés " [14].

Dans certains cas, les assecs durent jusqu'à l'hiver 1977-1978. Une pisciculture fonctionne plusieurs mois grâce au pompage dans un forage de secours.

Face à cette situation d'assèchement, le poisson est parfois transporté d'une rivière à une autre.

\subsection{La navigation fluviale}

\subsubsection{Les canaux}

La sévérité de l'étiage se traduit par deux types de conséquences sur l'alimentation en eau des canaux :

- les prises d'eau en rivières sont réduites au strict minimum, et quelquefois même stoppées;

- les réserves constituées en hiver dans les réservoirs, notamment ceux destinés à l'alimentation des biefs de partage, s'avèrent globalement déficitaires et en tout cas insuffisantes pour assurer une alimentation normale.

Il s'ensuit des mesures de restrictions à l'enfoncement des bateaux et même d'arrêt de navigation. Les décisions des chefs de service de navigation sont portées immédiatement à la connaissance des usagers par des avis à la batellerie. "Des dispositions sont prises pour que des bateaux ne se trouvent "piégés" sur des canaux sans issue " (cas des voies du Centre).

Enfin, là où la navigation reste possible, des consignes sont données aux éclusiers pour éviter les pertes d'eau : interdiction de "fausses bassinées " ${ }^{(6)}$, regroupage des bateaux pour un seul éclusage (bateaux de plaisance notamment) [8].

Lors des chômages (arrêts programmés de la navigation pour effectuer des travaux sur les ouvrages), il est recommandé de ne pas vider les biefs. En effet la vidange d'un bief en étiage sévère est généralement cause d'un retard important sur la reprise de la navigation après la fin du chômage.

(5) Les statistiques de la protection civile indiquent seulement 88000 ha.

(6) Une «fausse bassinée» est une mancuvre de portes d'écluse sans bateau dans le sas. 
Si la sécheresse de 1976 est à l'origine de la paralysie du trafic sur de nombreux canaux, il est des cas où elle a peu d'effets. Ainsi il n'y a pratiquement pas d'arrêts par manque d'eau sur le canal de la Marne à la Saône en 1976, oủ pourtant on en a noté auparavant :

- 5 jours consécutifs en 1947 du 23 au 29 décembre;

- 42 jours consécutifs en 1954 du 20 septembre au 30 octobre;

- 43 jours consécutifs en 1964 du 5 novembre au 17 décembre;

- 89 jours consécutifs en 1971/1972 du 15 novembre 1971 au 11 février 1972.

\subsubsection{Les rivières navigables}

La mauvaise hydraulicité entraîne une gêne de la navigation sur le Rhin. Les autres fleuves navigables canalisés enregistrent un trafic normal, même sur la Moselle où le caractère critique de la situation nécessite une nouvelle répartition de la ressource entre les différents usages, notamment au point critique de Flavigny-Messein.

Le débit de la Moselle à Flavigny tombe aux environs de $2 \mathrm{~m}^{3} / \mathrm{s}$. Le débit transité par le canal de l'Est, lui-même alimenté par la rigole de Flavigny est évalué à $2 \mathrm{~m}^{3} / \mathrm{s}$ également (voir figure 2). Après prélèvement des quantités nécessaires au District urbain de Nancy $\left(1,6 \mathrm{~m}^{3} / \mathrm{s}\right)$ et au canal de jonction entre le canal de l'Est et le canal de la Marne au Rhin, le débit de la Moselle est nul au barrage de Mereville. Les aciéries de Neuves-Maisons, s'alimentant d'ordinaire dans le canal dont le débit est insuffisant, mettent en œuvre une installation complémentaire de recyclage des eaux par la Moselle. Un recyclage externe est réalisé et la Moselle s'écoule vers l'amont au droit de l'aciérie. Les débits recyclés se montant à $1,5 \mathrm{~m}^{3} / \mathrm{s}$, il est probable que les températures s'accroissent à des niveaux inacceptables sur ce tronçon [11].

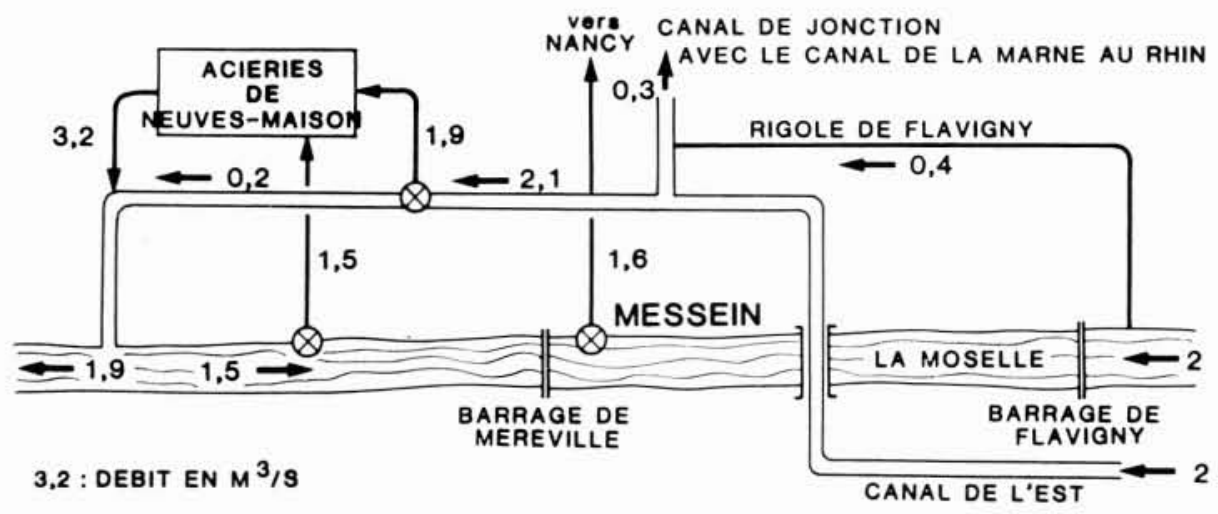

Figure 2. - Eté 1976 : Répartition des débits au point critique de Flavigny-Messein du bassin de la Moselle (d’après A.F.B.Rhin-Meuse).

\section{Conclusion}

La sécheresse de 1976 tire son caractère exceptionnel de la canicule de juin/juillet. La demande en eau croît de manière considérable et la part non restituée des prélèvements en rivière atteint sans doute un niveau record, dû en grande partie aux pompages d'irrigation. L'influence sur les débits d'étiage est telle qu'il est difficile de situer la sévérité de l'étiage parmi les millésimes les plus souvent cités $(1921,1947,1949,1954,1964)$, à conditions d'en connaître les débits. Par ailleurs, d’une région à l'autre, le classement est modifié.

Le secteur agricole compte les dommages les plus importants : l'appel à la solidarité nationale par la levée de l'impôt sécheresse traduit l'ampleur de la calamité. Cependant, dans tous les secteurs concernés de l'économie, les incidents les plus graves sont évités, soit que les équipements existants sont bien adaptés soit que la mobilisation de moyens exceptionnels et la réalisation d'urgence d'équipements de secours sont exécutés dans les plus brefs délais.

"Une vigilance et un dynamisme remarquable des pouvoirs publics " [10] se manifestent tant au niveau des services centraux des ministères, en centralisant et diffusant l'information et en autorisant des procédures exceptionnelles d'attribution de crédits, qu'au niveau départemental en déclenchant et en menant à bien des interventions rapides.

Le service des problèmes de l'eau du ministère de la qualité de la vie prend en charge la coordination et la diffusion de toutes les informations émanant de tous les ministères concernés par la sécheresse dans leurs compétences respectives. Outre ce rôle essentiel au sein de l'administration, il délivre aux média la substance des informations diffusées : ainsi il assume un rôle de conseiller technique dans l'émission d'Antenne 2 "Les dossiers 
de l'écran " consacrée le 20 juillet 1976 à la sécheresse et participe aux campagnes de sensibilisation par voie d'affiche.

On peut toujours faire mieux. L'analyse de la sécheresse de 1976 permet de replacer l'importance de l'eau dans l'économie des pays développés qu'on a trop tendance à croire suffisamment bien nantis pour en amortir les effets les plus néfastes.

Une première question vient à l'esprit : que se passe-t-il si une sécheresse type 76 dure au-delà de l'été, pendant l'automne et - pourquoi pas - une partie de l'hiver suivant? Certes, au service des barrages réservoirs du bassin de la Seine, la prudence veut que l'on garde de l'eau en réserve, au cas où l'étiage se poursuit. Il en résulte un stock de $30 \mathrm{Mm}^{3}$ inutilisé sur le barrage « Seine » en 1976 : peut-on faire autrement ? Vouloir répondre à cette question, c'est s'engager dans un programme d'études de longue haleine, encore en cours de réalisation. Résumer ce qui est réalisé dans ce domaine en 1984 dépasse le cadre de cet article. L'idée maitresse de ce programme d'études est l'amélioration de la connaissance de tous les processus susceptibles d'avoir une influence sur une gestion du réservoir adaptée à la demande et à la ressource disponible en rivière. La réussite éventuelle de cette démarche contribuera à mieux programmer les équipements à venir...
Cet exemple complète, sans les contredire, les orientations de la politique de l'eau esquissées encore à chaud, juste après la sécheresse de 1976 par J.F. Saglio :

" - poursuivre l'effort de connaissance des phénomènes hydrauliques notamment en perfectionnant les réseaux de mesure;

- adopter une politique de la sécurité de l'approvisionnement en eau, en particulier des collectivités publiques; - améliorer les ressources en eau par la construction de réserves d'eau superficielles pour l'alimentation en eau potable, l'irrigation, les besoins industriels et l'amélioration de la qualité;

- rationaliser l'utilisation des ressources en eau par une exploitation judicieuse des nappes souterraines, impliquant notamment une protection renforcée de celles-ci; - promouvoir auprès des usagers une politique d'utilisation de l'eau évitant les gaspillages " [12].

Nous sommes en 1984. En huit ans beaucoup de choses évoluent. Des équipements nouveaux sont réalisés ou en cours de réalisation. La demande en eau subit des modifications importantes. La qualité de l'eau des rivières et des nappes évolue elle aussi.

Comme il est peu probable que la prochaine sécheresse exceptionnelle soit identique à celle de 1976, tout porte à croire que le déroulement de la lutte contre ses effets comportera de nombreuses différences.

\section{PRINCIPALES SOURCES DE DOCUMENTATION}

A - Notes sur la sécheresse 1976 du Service des problèmes de l'eau. Direction de la prévention des pollutions et nuisances. Secrétariat d'Etat à l'environnement et à la qualité de la vie.

1 - La sécheresse au 31 mai 19766 pages + figures + annexes

2 - Situation au 10 juin 3 pages + figures + nombreuses annexes. Réunions du 15 juin 1975 du groupe interministériel sur les problèmes de sécheresse

3 - La sécheresse au 30 juin 1976, 14 pages + figures +2 annexes

4 - La sécheresse au 15 juillet 1976, 13 pages + annexes

5 - La sécheresse au 31 juillet 1976, 15 pages + tableaux et figures

6 - La sécheresse au 15 août 1976, 3 pages + figures + nombreuses annexes

7 - La sécheresse au 31 août 1976, 3 pages + annexes

8 - La sécheresse 1976 - Aspects, conséquences et enseignements à en tirer,27 pages dactylographiées non daté

8 bis - Mission Interministérielle de l'eau - La sécheresse 1976, aspects climatologiques, conséquences, actions administratives, propositions. 40 pages non daté.

9 - Mission Interministérielle de l'eau - réunion du 26 août 1982. Situation de la sécheresse $1982-4$ pages +6 pièces annexes

\section{B - Notes des Agences financières de bassin}

10 - A.F.B. Adour-Garonne - La sécheresse de 1976 dans le bassin Adour-Garonne, 20 pages + annexes - novembre 1976

11 - A.F.B. Rhin-Meuse, La sécheresse de 1976 dans le bassin Rhin-Meuse, 25 pages +15 figures

\section{$\mathrm{C}$ - Articles dans les revues}

Revue "Seine-Normandie " spécial sécheresse - décembre 1976

12 - J.F. SAGLIO. - Editorial pages 2 et 3

13 - J. CARLIER. - Les conséquences de la sécheresse 1976 pour l'agriculture dans le bassin "SeineNormandie ", pages 12 à 16

14 - M. Mallly. - A propos de la sécheresse... le point de vue des pêcheurs, pages 17 à 21

15 - J.L. Jourdet. - Les conséquences de la sécheresse pour Electricité de France, pages 22 à 24

16. - XXX. - La distribution d'eau en région parisienne pendant la sécheresse en 1976, pages 25 à 30

17 - M. Odier. - L'incidence de la sécheresse sur la qualité de l'eau de Seine à Paris, pages 31 à 35

Revue PCM " Sécheresse : le bilan "

18 - J.D. SAGLio. - La sécheresse de 1976, page 16

19 - A. Goubet. - Les problèmes de la production électrique, pages 17 à 19 
20 - F. Bourgin. - Le département du Finistère, pages 20 à 22

21 - XXX. - L'aide apportée par l'armée, pages 23 à 26

22 - F. VALIRON. - L'eau en région parisienne, pagẹs 27 à 33

23 - G. Castany et J. Margat. - Les eaux souterraines, pages 34 à 39

24 - B. VERDON. - Le département de l'Allier, pages 40 à 44

Techniques et sciences municipales - La sécheresse de 1976

25 - M. DARGent. - Les aspects quantitatifs, no 8 et 9 , pages 343 à 350

26 - M. Rapinat. - Effet de la sécheresse sur la qualité de l'eau, no 8 et 9 , pages 351 à 357

27 - J. Prompsy Réalimentation des nappes n०10, pages 413 à 420

D. Statistiques, notes et publications des Ministères de l'Agriculture, de l'Intérieur et du Commissariat aux risques majeurs.

28 - Statistiques sur les feux de forêts 1976, 16 pages 1977 et 1978,34 pages
1979, 20 pages

1980, 21 pages

1981, 23 pages

1982, 23 pages

1983, en préparation

Ministère de l'agriculture. Direction de l'aménagement. Service de l'aménagement rural et des équipements

29 - «Les conséquences d'une sécheresse sur l'alimentation en eau potable : celle de 1976 " - 18 pages dactylographiées

\section{Bulletin d'Information du Ministère de l'agriculture}

30 - no 733 du 3.07.1976, Ch. Bonnet fait le point sur les problèmes de la sécheresse pages 21 à 25

31 - no 738 du 4.09.1976, Acompte aux agriculteurs victimes de la sécheresse pages 17 à 21

$32-$ no 741 du 25.09.1976, Aide exceptionnelle aux agriculteurs victimes de la sécheresse pages 23 à 27

33 - Rapport au Président de la République présenté par Haroun Tazieff, Commissaire à l'étude et à la prévention des risques naturels majeurs, 58 pages.

Journal officiel de la République française 1983.

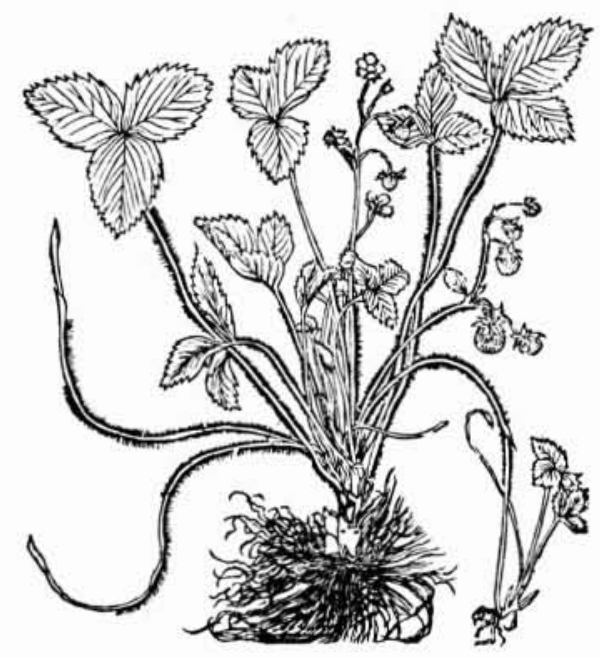




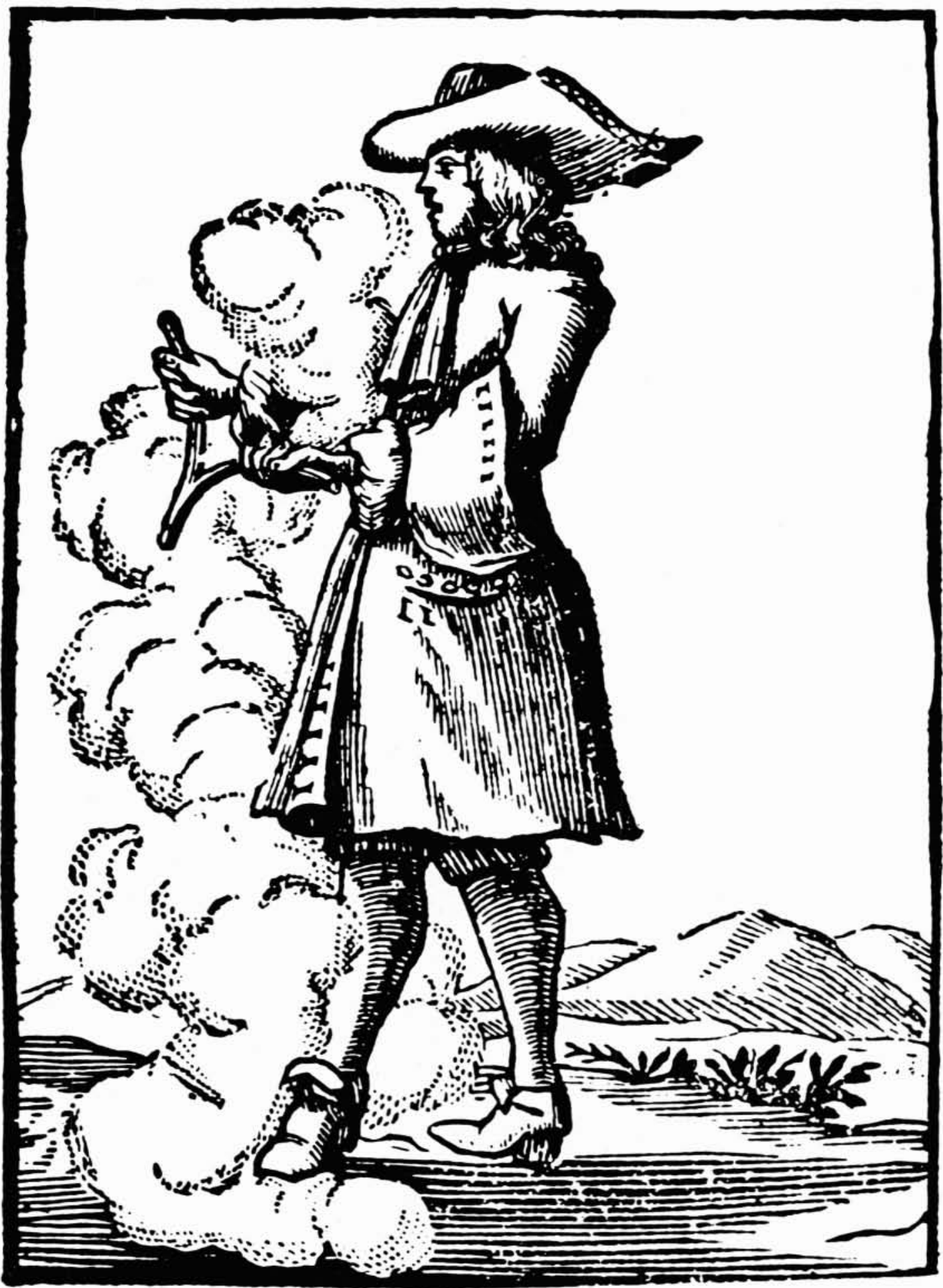

